\title{
Translational control of mRNAs by 3'-Untranslated region binding proteins
}

\author{
Akio Yamashita, ${ }^{1, *}$ Osamu Takeuchi ${ }^{2, *}$ \\ ${ }^{1}$ Department of Molecular Biology, Yokohama City University School of Medicine, Yokohama 236-0004, ${ }^{2}$ Laboratory of Infection and \\ Prevention, Institute for Frontier Life and Medical Sciences, Kyoto University, Kyoto 606-8507, Japan
}

\begin{abstract}
Eukaryotic gene expression is precisely regulated at all points between transcription and translation. In this review, we focus on translational control mediated by the $3^{\prime}$-untranslated regions (UTRs) of mRNAs. mRNA 3'-UTRs contain cis-acting elements that function in the regulation of protein translation or mRNA decay. Each RNA binding protein that binds to these cis-acting elements regulates mRNA translation via various mechanisms targeting the mRNA cap structure, the eukaryotic initiation factor $4 \mathrm{E}$ (elF4E)-elF4G complex, ribosomes, and the poly (A) tail. We also discuss translation-mediated regulation of mRNA fate. [BMB Reports 2017; 50(4): 194-200]
\end{abstract}

\section{INTRODUCTION}

Translational regulation of mRNA is an immediate and precise mechanism to control gene expression in various biological processes, including development, differentiation, and responses to extracellular stress. Global quantification analysis indicates that the cellular abundance of proteins in mammals is predominantly controlled at the level of translation (1). In vivo, mRNAs do not exist as bare mRNA molecules but as mRNA-protein complexes with RNA-binding proteins (RBPs) (2-5). More than one thousand RBPs have been identified, and they bind to specific cis-acting elements, consisting of sequence elements, stem-loop structures and/or modified nucleotides (6-8). For many genes, alternative poly $(\mathrm{A})$ addition and alternative splicing give rise to $3^{\prime}$-UTR variants (Fig. 1A and $\mathrm{B})$. These variants are controlled by specific posttranscriptional regulation $(9,10)$.

The cap-dependent mRNA translational process is divided

${ }^{*}$ Corresponding authors. Akio Yamashita, Tel: +81-45-787-2598; Fax: +81-45-785-4140; E-mail: yamasita@yokohama-cu.ac.jp, Osamu Takeuchi, Tel: +81-75-751-4024; Fax: +81-75-761-5766; E-mail: otake@virus.kyoto-u.ac.jp

https://doi.org/10.5483/BMBRep.2017.50.4.040

Received 8 March 2017

Keywords: RNA binding protein, Translation, Translation initiation, 3'-UTR into three major steps: initiation, elongation, and termination. Each step is elaborately regulated by multiple mRNA 3'-UTR binding proteins in a cell type- and species-specific manner $(11,12)$. In this review, we present examples of RBP-mediated regulation of translation and we discuss their biological roles.

\section{MOLECULAR MECHANISM OF CAP-DEPENDENT mRNA TRANSLATION BY 3'-UTR BINDING PROTEINS}

In eukaryotes, most protein coding mRNAs have a $5^{\prime}$-terminal cap structure and a 3'-terminal poly-adenine. Histone mRNAs, however, are an exception, having a specific stem-loop structure in the 3 '-terminal region (13). The cap structure acts as an anchor and is critical for translation initiation by the eukaryotic initiation complex (14). In eukaryotes, this capdependent translation initiation is implemented by two macromolecular complexes, namely, the eukaryotic initiation factor-4F (elF4F) complex, consisting of elF4E, elF4G and elF4A, and the $43 \mathrm{~S}$ initiation complex, consisting of a $40 \mathrm{~S}$ ribosome, elF3, elF1A, elF2 and methionyl-tRNA $(11,12)$. The elF4F complex connects the $5^{\prime}$ - and 3 '-termini of the mRNA via interactions with poly $(\mathrm{A})$ binding protein $(\mathrm{PABP})$ or histone stem-loop binding protein (SLBP) (13). This mRNA circularization plays a significant role in efficient translation, probably by accelerating ribosome recycling (Fig. 2A) $(11,12)$. In addition, eukaryotic release factor 3 (eRF3) directly binds to the PABP/elF4G complex and stimulates translation (15). Hence, mRNA translation can be modulated by the cap-binding protein complex. The circularization of mRNA is also a significant molecular feature in the regulation of cap-dependent mRNA translation by 3'-UTR RBPs $(11,12)$.

\section{REGULATION OF TRANSLATION THROUGH THE CAP STRUCTURE (Fig. 2B)}

The cap-binding protein, elF4E, is the foundation of a translation initiation complex at the $5^{\prime}$-terminal cap structure $(11,12)$. In addition, the elF4E-related molecule, 4E homologous protein (4EHP), competitively and directly binds the cap structure. However, 4EHP represses translation because of its weak affinity to elF4G (16). 4EHP can be recruited by RBPs such as Bicoid (which binds to a specific cis-element in the 


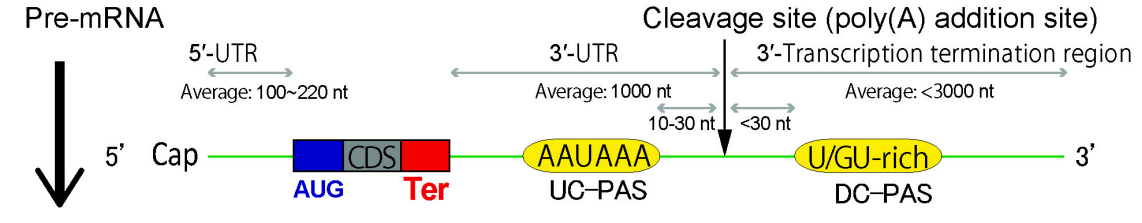

mRNA

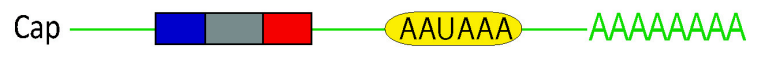

B
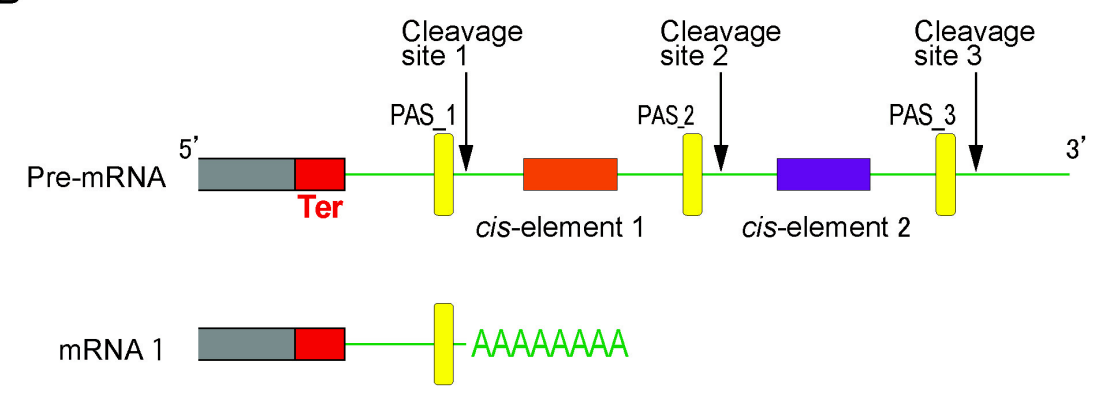

$3^{\prime}$-UTR

mRNA 2

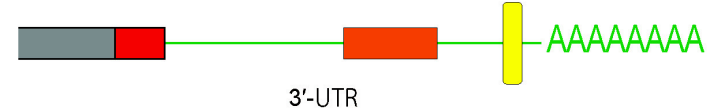

mRNA 3

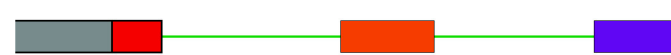

3'-UTR
Fig. 1. mRNA processing generates 3'-UTR variants. (A) Schematic depictions of pre-mRNA and mature mRNA. UTR (untranslated region), CDS (protein coding sequence), UC-PAS (upstream core polyadenylation signal), DC-PAS (downstream core polyadenylation signal). (B) mRNA isoforms with alternative poly $(\mathrm{A})$ additions.
3'-UTR of caudal mRNA) and the Pumilio/Nanos/Brat complex (which binds to a specific cis-element in the 3'-UTR of hunchback mRNA) during Drosophila melanogaster development $(17,18)$. Mammalian $4 \mathrm{EHP}$ also has the ability to suppress translation via the same mechanisms $(19,20)$. On the other hand, 4EHP can augment translation during hypoxia in human U87MG glioblastoma cells (21). The transcription factor, hypoxia-Inducible Factor $2 \alpha$ (HIF-2 $\alpha$ ), can bind both DNA and RNA and forms a complex with RBM4 on the 3'-UTRs of a subset of mRNAs including FGFR mRNA. The HIF-2 $\alpha /$ RBM 4 complex then recruits $4 \mathrm{EHP}$ to stimulate translation. elF4A, but not elF4G, is present in this complex (21).

\section{REGULATION OF TRANSLATION THROUGH THE elF4E-elF4G STRUCTURE (Fig. 2C)}

The interaction between elF4E and elF4G is required for mRNA circularization and the initiation of cap-dependent translation $(11,12)$. D. melanogaster Cup, Xenopus laevis Maskin, mammalian Neuroguidin (NGD) and mammalian cytoplasmic fragile $X$ mental retardation protein (FMRP) interacting protein 1 (CYFIP1) bind elF4E competitively with elF4G and repress translation. These translational repressors are recruited to mRNAs through specific 3'-UTR binding proteins, namely, Cup/Bruno, Cup/Smaug, Maskin/cytoplasmic polyadenylation element binding protein (CPEB), NGD/CPEB, and CYFIP1/FMRP (22-25).

microRNAs (miRNAs) are small RNA molecules consisting of 21-24 nucleotides that form microRNA-induced silencing complexes (miRISCs) with Argonaute (Ago) proteins and repress translation. Ago proteins have isoform specific mechanisms for repression of translation. In D. melanogaster, after miRISC binds to a miRNA target site in an mRNA 3'-UTR, Ago2 represses translation by competing for elF4E binding, which is similar to Cup (26). 
A

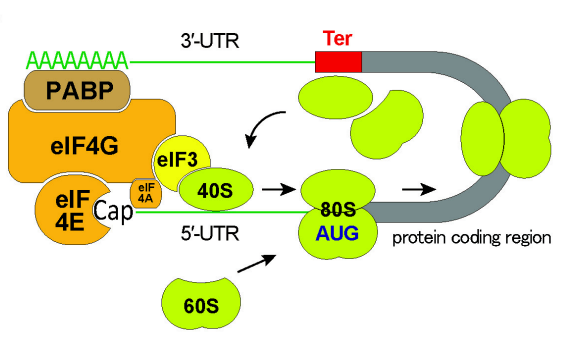

B

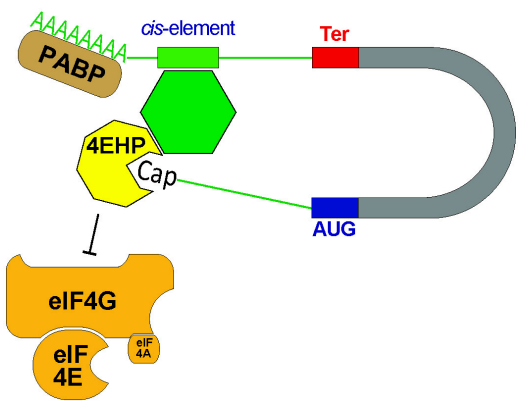

D

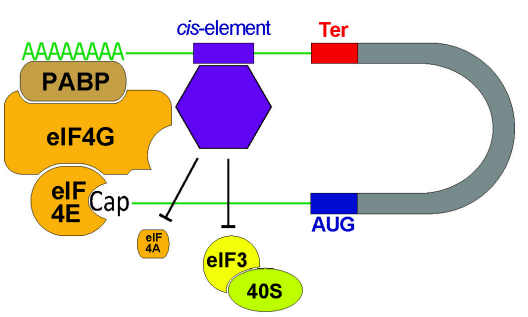

435 initiation complex

$\mathrm{F}$

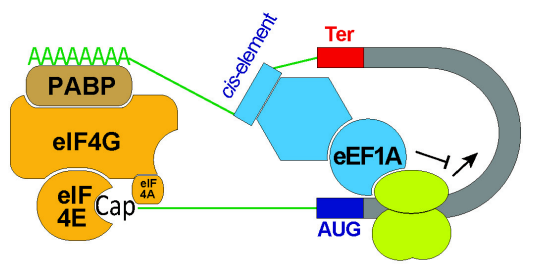

C

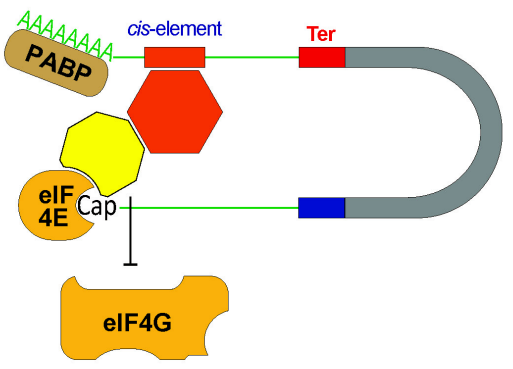

$\mathrm{E}$

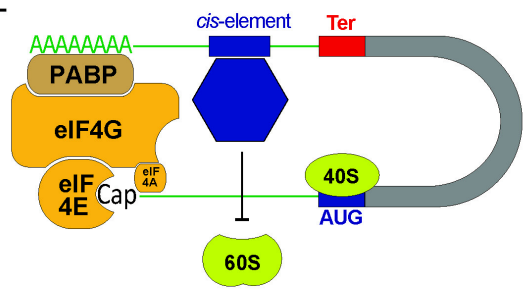

Fig. 2. Translational control by $3^{\prime}-$ UTR binding proteins. (A) Schematic depiction of circularized mRNA translation. (B) Regulation of translation through the cap structure. (C) Regulation of translation through the elF4E-elF4G complex. (D) Regulation of translation through the 43S translation initiation complex. (E) Regulation of translation through the 80S ribosome assembly. (F) Regulation of translation through translation elongation. (G) Regulation of translation through the poly(A) tail.

\section{REGULATION OF TRANSLATION THROUGH THE 435 TRANSLATION INITIATION COMPLEX (Fig. 2D)}

In contrast to D. melanogaster Ago2, D. melanogaster Ago1 and vertebrate Ago2 repress translation by interfering with the assembly of a functional elF4F complex. This occurs through the displacement of elF4A (in vertebrate elF4A1 and elF4A2) from the $m R N A$, leading to the functional suppression of the $43 \mathrm{~S}$ initiation complex $(27,28)$. A conflicting model of vertebrate Ago2 action has been proposed. In this model, the vertebrate Ago-associated CCR4-NOT complex recruits elF4A2. The elF4A2 would then inhibit translation initiation by preventing the recruitment of active elF4A1 (29). Further investigations are necessary to verify these models $(30,31)$. In addition, these Ago isoforms are expected to affect mRNA circularization through complex formation with trinucleotide repeat-containing protein 6 (TNRC6), which has the ability to repress translation $(30,31)$.

The gamma interferon-activated inhibitor of translation (GAIT) complex consists of a ribosomal protein L13a (rpL13a), 
glyceraldehyde-3-phosphate dehydrogenase (GAPDH), glutamylprolyl-tRNA synthetase (EPRS), and NS1-associated protein-1 (NSAP1), and represses translation initiation by inhibiting recruitment of the 435 translation initiation complex. The GAIT complex recognizes specific stem-loop structure elements and, in response to interferon- $\gamma$, forms on a subset of $3^{\prime}$-UTR elements of mRNAs, including those of ceruloplasmin and vascular endothelial growth factor (VEGF). mRNA circularization is maintained during GAIT-mediated repression of translation and is expected to contribute to the action of the 3'-UTR binding protein complex on 5'-terminal cap-dependent translation initiation (32).

During female embryonic development in $D$. melanogaster, translation of mls2 mRNA is prevented by the Sex-lethal (SXL)/upstream of N-ras (UNR) complex formed on a specific cis-acting element in the $3^{\prime}$-UTR of $\mathrm{mls} 2 \mathrm{mRNA}$. The SXL/UNR complex inhibits the recruitment of the 43S translation initiation complex by maintaining mRNA circularization (33). In addition, SXL binds to the $5^{\prime}$-UTR of m/s2 mRNA and represses initiation codon scanning by the $43 \mathrm{~S}$ initiation complex in an upstream open reading frame (uORF)-dependent manner (34).

Emerging evidence indicates that base modifications, including inosine, N6-methyladenosine $\left(\mathrm{m}^{6} \mathrm{~A}\right)$, N1-methyladenosine, 5-methylcytosine, 5-hydroxymethylcytidine, and pseudouridine, can modulate the fate of mRNA (35). Among these modifications, $\mathrm{m}^{6} \mathrm{~A}$ in $3^{\prime}$-UTRs promotes translation when recognized by $\mathrm{YTH}$ domain-containing family protein 1 (YTHDF1) (36). Similarly, methyltransferase like 3 (METTL3), a catalytic subunit of the $\mathrm{m}^{6} \mathrm{~A}$ methyltransferase complex, recognizes an un-methylated site of $\mathrm{m}^{6} \mathrm{~A}$ and promotes translation (37). Although the precise mechanism needs to be elucidated, both YTHDF1 and METTL3 form a complex with components of the $43 \mathrm{~S}$ translation initiation complex. Intriguingly, YTHDF2, another $\mathrm{m}^{6} \mathrm{~A}$ decoder, competitively binds the same site and degrades mRNA (38). These complementary functions of $\mathrm{m}^{6} \mathrm{~A}$ decoders could enable dynamic and precise regulation of gene expression.

\section{REGULATION OF TRANSLATION THROUGH $80 \mathrm{~S}$ RIBOSOME ASSEMBLY (Fig. 2E)}

During the differentiation of erythrocytes, the heterogeneous nuclear ribonucleic protein $\mathrm{K}(\mathrm{hnRNPK}) / \mathrm{hnRNPE} 1$ complex associates with a specific cis-acting element in the $3^{\prime}$-UTR of 15-lipoxygenase mRNA. The hnRNPK/hnRNPE1 complex represses translation by inhibiting $60 \mathrm{~S}$ ribosome binding to the $43 \mathrm{~S}$ initiation complex and assembly of the 80S ribosome (39).

Translational repression during mRNA targeting coupled to local translation is essential for spatial restriction of protein production (40). In Saccharomyces cerevisiae, ASH1 mRNA, which encodes a repressor of mating-type switching, localizes to the tip of daughter cell. Translation of localizing ASH1 mRNA is silenced by Pumilio-homology domain protein 6 protein (Puf6p). Puf6p prevent assembly of $80 \mathrm{~S}$ ribosomes on ASH1 mRNA. Puf6p recognizes a cis-acting element in the 3'-UTR of ASH1 mRNA and binds yeast elF5B. elF5B is an essential component of $80 \mathrm{~S}$ ribosome assembly; therefore, this RNA-dependent interaction is essential for translational repression of the ASH1 mRNA. Intriguingly, casein kinase 2 (CK2)-mediated phosphorylation of Puf6p restores ASH1 mRNA translation after mRNA localization (41).

In mammals, Z-DNA-binding protein 1 (ZBP1) represses $\beta$-actin mRNA translation through the inhibition of $80 \mathrm{~S}$ assembly before its localization to the leading edge of cell migration. After mRNA localization, Src kinase-mediated phosphorylation of ZBP1 restores $\beta$-actin mRNA translation (42).

\section{REGULATION OF TRANSLATION THROUGH ELONGATION (Fig. 2F)}

Phosphorylation-mediated regulation of translation through repression of RBPs plays a critical checkpoint coordinating the expression of transforming growth factor $\beta$ (TGF $\beta$ )-induced epithelial-mesenchymal transition (EMT) transcripts in tumorigenesis and metastatic progression. The hnRNPE1/eukaryotic elongation factor $1 \mathrm{~A}$ (eEF1A) complex forms on specific cis-acting elements in the $3^{\prime}$-UTR of disabled-2 mRNA and interleukin-like EMT inducer (ILEI) mRNA. The hnRNPE1/eEF1A complex represses translation by associating with the translating $80 \mathrm{~S}$ ribosome to "stall" on mRNA by preventing eEF1A dissociation from the $80 \mathrm{~S}$ ribosome. Akt2, which is activated by TGF $\beta$ signaling, mediates phosphorylation of hnRNPE1 and induces hnRNPE1 dissociation from mRNA, thereby restoring translation of target mRNAs. Importantly, attenuation of hnRNP E1 expression induced EMT and enabled cells to form metastatic lesions in vivo (43).

eEF1A dissociation-mediated translational repression is also used by Caenorhabditis elegans gld-1 mRNA. The FBF-1 (nematode Pumilio)/CSR-1 (Ago isoform)/EFT-3 (nematode eEF1) complex forms on a specific cis-acting element in the $3^{\prime}-\mathrm{UTR}$ of gld-1 mRNA and represses translation (44).

\section{REGULATION OF TRANSLATION THROUGH THE POLY(A) TAIL (Fig. 2G)}

Many RBPs bound to 3'-UTRs of mRNAs induce mRNA degradation, although we do not discuss this in detail in this review (10). These RBPs associate with the deadenylase complex (e.g. poly(A)-specific ribonuclease (PARN), the poly(A) ribonuclease 2 (PAN2)/PAN3 complex and the carbon catabolite repression 4 (CCR4)-negative on TATA-less (NOT) complex) to enhance deadenylation (45). The terminal uridylyl transferases, TUT4 and TUT7, selectively recognize and catalyze uridylation of deadenylated mRNAs with short A-tails ( $<25$ nucleotides). This oligo uridylation enhanced the further degradation of the mRNA body (46).

Deadenylation is thought to dissociate PABP from the $3^{\prime}$-tail 
of mRNA and to disrupt mRNA circularization, thereby repressing translation. However, recent studies demonstrated that median poly(A) lengths are about 60-100 nucleotides (nt), which is shorter than the 150-200 nt thought to be typical of mammalian poly(A) tails. Poly(A) lengths of $>20$ nt are not correlated with translational efficiency in somatic cells (47, 48). When the poly (A) tail length is $<20 \mathrm{nt}$, translation is repressed in most genes in somatic cells (48). Because PABP binds to poly(A)s of 20 nt lengths, one PABP molecule might be sufficient to support mRNA circularization and poly(A)-dependent efficient translation.

In contrast to somatic cells, poly(A) tail length and translational efficiency are coupled in embryonic cells (47, 49). In X. laevis oocytes, cytoplasmic polyadenylation element binding protein (CPEB) binds specific cis-acting elements in the 3'-UTRs of mRNAs encoding cell cycle-related proteins, such as cyclin B. CPEB recruits PARN deadenylase and short poly(A) mRNAs are stabilized before oocyte maturation (50, 51). The stimulation of oocyte maturation induces CPEB phosphorylation and promotes the association of cleavage and polyadenylation specificity factor (CPSF) and Germ Line Development 2 (GLD-2), poly (A) polymerase. Poly(A) elongation then augments translation of target mRNAs.

\section{REGULATION OF MRNA DECAY THROUGH TRANSLATION}

As mentioned above, the $3^{\prime}$-UTR plays a key role in translational control. However, reciprocally, translation also regulates 3'-UTR-mediated mRNA decay. For instance, when exon-junction complexes are bound to the 3'-UTR, premature translation termination is recognized, and the mRNA is degraded by the nonsense-mediated mRNA decay pathway which acts as an mRNA quality control system (52). Normal translation termination codon recognition also stimulates mRNA degradation of inflammation-related mRNAs containing a specific stem-loop in the $3^{\prime}$-UTR. In this case, the stem-loop is recognized by Regnase-1, an endonuclease (53). Similarly, translational termination induces the degradation of replicationdependent histone mRNAs and Staufen1-mediated mRNA decay $(54,55)$. An RNA helicase called Up frame shift 1 (UPF1) is required for the translation-dependent mRNA decay systems described above. Taken together, 3'-UTRs can act bi-directionally in translational regulation and mRNA decay mechanisms that are closely involved with each other.

\section{PERSPECTIVES}

In the present review, we briefly introduced the mechanism of translational control by mRNA 3'-UTR-binding proteins. Translational control is recognized as an essential regulatory mechanism of gene expression in various biological processes. For example, interferon- $\gamma$ production from T-cells is regulated by translational control coupled with glycolysis. GAPDH, an enzyme essential for glycolysis, also acts as an RBP that directly binds to a cis-acting element in the $3^{\prime}$-UTR of the interferon- $\gamma$ mRNA and acts as a translational repressor in inactive $\mathrm{T}$ cells. $\mathrm{T}$ cell activation drastically alters the metabolic status of $\mathrm{T}$ cells, with aerobic glycolysis promoted over oxidative phosphorylation, and GAPDH can dissociate from the interferon- $\gamma$ mRNA to function as an aerobic glycolysis enzyme. In this situation, GAPDH no longer represses the translation of interferon- $\gamma$ mRNA, leading to an increase in interferon- $\gamma$ production (56).

Many RBPs involved in translational control and/or mRNA degradation have additional roles in alternative pre-mRNA splicing, alternative poly(A) addition and other mRNA processing events (57). In addition, analysis of translational control using plasmid vector-based reporter assays must consider the possibility of unexpected/undesired transcription from all regions of the circular plasmid DNA and unexpected processing of mRNA. Hence, in vitro reconstituted translation experiments should also be performed in addition to cell and/or animal-based analysis.

\section{CONFLICTS OF INTEREST}

The authors have no conflicting financial interests.

\section{REFERENCES}

1. Schwanhausser B, Busse D, Li N et al (2011) Global quantification of mammalian gene expression control. Nature 473, 337-342

2. Kong J and Lasko P (2012) Translational control in cellular and developmental processes. Nat Rev Genet 13, 383-394

3. Moore MJ (2005) From birth to death: the complex lives of eukaryotic mRNAs. Science 309, 1514-1518

4. Jung H, Gkogkas CG, Sonenberg N and Holt CE (2014) Remote control of gene function by local translation. Cell $157,26-40$

5. Ivanov P and Anderson P (2013) Post-transcriptional regulatory networks in immunity. Immunol Rev 253, 253-272

6. Castello A, Fischer B, Frese CK et al (2016) Comprehensive Identification of RNA-Binding Domains in Human Cells. Mol Cell 63, 696-710

7. Castello A, Fischer B, Eichelbaum $K$ et al (2012) Insights into RNA biology from an atlas of mammalian mRNAbinding proteins. Cell 149, 1393-1406

8. Gerstberger S, Hafner M and Tuschl T (2014) A census of human RNA-binding proteins. Nat Rev Genet 15, 829-845

9. Tian B and Manley JL (2017) Alternative polyadenylation of mRNA precursors. Nat Rev Mol Cell Biol 18, 18-30

10. Chen CA and Shyu AB (2017) Emerging Themes in Regulation of Global mRNA Turnover in cis. Trends Biochem Sci 42, 16-27

11. Sonenberg $N$ and Hinnebusch AG (2009) Regulation of translation initiation in eukaryotes: mechanisms and biological targets. Cell 136, 731-745

12. Jackson RJ, Hellen CU and Pestova TV (2010) The 
mechanism of eukaryotic translation initiation and principles of its regulation. Nat Rev Mol Cell Biol 11, 113-127

13. Danckwardt S, Hentze MW and Kulozik AE (2008) 3' end mRNA processing: molecular mechanisms and implications for health and disease. EMBO J 27, 482-498

14. Furuichi $Y$ (2015) Discovery of m(7)G-cap in eukaryotic mRNAs. Proc Jpn Acad Ser B Phys Biol Sci 91, 394-409

15. Uchida $N$, Hoshino $S$, Imataka $H$, Sonenberg $N$ and Katada T (2002) A novel role of the mammalian GSPT/eRF3 associating with poly(A)-binding protein in Cap/Poly(A)-dependent translation. J Biol Chem 277, 50286-50292

16. Rom E, Kim HC, Gingras AC et al (1998) Cloning and characterization of 4EHP, a novel mammalian elF4Erelated cap-binding protein. J Biol Chem 273, 1310413109

17. Cho PF, Poulin F, Cho-Park YA et al (2005) A new paradigm for translational control: inhibition via 5'-3' mRNA tethering by Bicoid and the elF4E cognate 4EHP. Cell 121, 411-423

18. Cho PF, Gamberi C, Cho-Park YA, Cho-Park IB, Lasko P and Sonenberg N (2006) Cap-dependent translational inhibition establishes two opposing morphogen gradients in Drosophila embryos. Curr Biol 16, 2035-2041

19. Morita M, Ler LW, Fabian MR et al (2012) A novel 4EHP-GIGYF2 translational repressor complex is essential for mammalian development. Mol Cell Biol 32, 35853593

20. Fu R, Olsen MT, Webb K, Bennett EJ and Lykke-Andersen J (2016) Recruitment of the 4EHP-GYF2 cap-binding complex to tetraproline motifs of tristetraprolin promotes repression and degradation of mRNAs with AU-rich elements. RNA 22, 373-382

21. Uniacke J, Holterman CE, Lachance G et al (2012) An oxygen-regulated switch in the protein synthesis machinery. Nature 486, 126-129

22. Nelson MR, Leidal AM and Smibert CA (2004) Drosophila Cup is an elF4E-binding protein that functions in Smaugmediated translational repression. EMBO J 23, 150-159

23. Stebbins-Boaz B, Cao Q, de Moor $\mathrm{CH}$, Mendez $\mathrm{R}$ and Richter JD (1999) Maskin is a CPEB-associated factor that transiently interacts with elF-4E. Mol Cell 4, 1017-1027

24. Jung MY, Lorenz L and Richter JD (2006) Translational control by neuroguidin, a eukaryotic initiation factor $4 \mathrm{E}$ and CPEB binding protein. Mol Cell Biol 26, 4277-4287

25. Napoli I, Mercaldo V, Boyl PP et al (2008) The fragile X syndrome protein represses activity-dependent translation through CYFIP1, a new 4E-BP. Cell 134, 1042-1054

26. Iwasaki S, Kawamata T and Tomari Y (2009) Drosophila argonaute1 and argonaute2 employ distinct mechanisms for translational repression. Mol Cell 34, 58-67

27. Fukaya T, Iwakawa HO and Tomari Y (2014) MicroRNAs block assembly of elF4F translation initiation complex in Drosophila. Mol Cell 56, 67-78

28. Fukao A, Mishima Y, Takizawa N et al (2014) MicroRNAs trigger dissociation of elF4AI and elF4All from target mRNAs in humans. Mol Cell 56, 79-89

29. Meijer HA, Kong YW, Lu WT et al (2013) Translational repression and elF4A2 activity are critical for microRNA- mediated gene regulation. Science 340, 82-85

30. Filipowicz W and Sonenberg N (2015) The long unfinished march towards understanding microRNA-mediated repression. RNA 21, 519-524

31. Izaurralde $E$ (2013) A role for elF4All in microRNAmediated mRNA silencing. Nat Struct Mol Biol 20, 543-545

32. Mukhopadhyay R, Jia J, Arif A, Ray PS and Fox PL (2009) The GAIT system: a gatekeeper of inflammatory gene expression. Trends Biochem Sci 34, 324-331

33. Duncan K, Grskovic M, Strein C et al (2006) Sex-lethal imparts a sex-specific function to UNR by recruiting it to the msl-2 mRNA 3' UTR: translational repression for dosage compensation. Genes Dev 20, 368-379

34. Medenbach J, Seiler M and Hentze MW (2011) Translational control via protein-regulated upstream open reading frames. Cell 145, 902-913

35. Harcourt EM, Kietrys AM and Kool ET (2017) Chemical and structural effects of base modifications in messenger RNA. Nature 541, 339-346

36. Wang X, Zhao BS, Roundtree IA et al (2015) N(6)methyladenosine Modulates Messenger RNA Translation Efficiency. Cell 161, 1388-1399

37. Lin S, Choe J, Du P, Triboulet R and Gregory RI (2016) The m(6)A Methyltransferase METTL3 Promotes Translation in Human Cancer Cells. Mol Cell 62, 335-345

38. Wang X, Lu Z, Gomez A et al (2014) N6-methyladenosinedependent regulation of messenger RNA stability. Nature $505,117-120$

39. Ostareck DH, Ostareck-Lederer A, Wilm M, Thiele BJ, Mann M and Hentze MW (1997) mRNA silencing in erythroid differentiation: hnRNP K and hnRNP E1 regulate 15-lipoxygenase translation from the 3' end. Cell 89, 597-606

40. Besse F and Ephrussi A (2008) Translational control of localized mRNAs: restricting protein synthesis in space and time. Nat Rev Mol Cell Biol 9, 971-980

41. Deng Y, Singer RH and Gu W (2008) Translation of ASH1 mRNA is repressed by Puf6p-Fun12p/elF5B interaction and released by CK2 phosphorylation. Genes Dev 22, 1037-1050

42. Huttelmaier S, Zenklusen D, Lederer M et al (2005) Spatial regulation of beta-actin translation by Src-dependent phosphorylation of ZBP1. Nature 438, 512-515

43. Hussey GS, Chaudhury A, Dawson AE et al (2011) Identification of an mRNP complex regulating tumorigenesis at the translational elongation step. Mol Cell 41, 419-431

44. Friend K, Campbell ZT, Cooke A, Kroll-Conner P, Wickens MP and Kimble J (2012) A conserved PUF-AgoeEF1A complex attenuates translation elongation. Nat Struct Mol Biol 19, 176-183

45. Goldstrohm AC and Wickens M (2008) Multifunctional deadenylase complexes diversify mRNA control. Nat Rev Mol Cell Biol 9, 337-344

46. Lim J, Ha M, Chang H et al (2014) Uridylation by TUT4 and TUT7 marks mRNA for degradation. Cell 159, 1365-1376

47. Subtelny AO, Eichhorn SW, Chen GR, Sive H and Bartel DP (2014) Poly(A)-tail profiling reveals an embryonic 
switch in translational control. Nature 508, 66-71

48. Park JE, Yi H, Kim Y, Chang H and Kim VN (2016) Regulation of Poly(A) Tail and Translation during the Somatic Cell Cycle. Mol Cell 62, 462-471

49. Lim J, Lee M, Son A, Chang $H$ and Kim VN (2016) mTAIL-seq reveals dynamic poly(A) tail regulation in oocyte-to-embryo development. Genes Dev 30, 16711682

50. Kim JH and Richter JD (2006) Opposing polymerasedeadenylase activities regulate cytoplasmic polyadenylation. Mol Cell 24, 173-183

51. Hake LE and Richter JD (1994) CPEB is a specificity factor that mediates cytoplasmic polyadenylation during Xenopus oocyte maturation. Cell 79, 617-627

52. Schweingruber C, Rufener SC, Zund D, Yamashita A and Muhlemann O (2013) Nonsense-mediated mRNA decay mechanisms of substrate mRNA recognition and degradation in mammalian cells. Biochim Biophys Acta 1829,
612-623

53. Mino T, Murakawa Y, Fukao A et al (2015) Regnase-1 and Roquin Regulate a Common Element in Inflammatory mRNAs by Spatiotemporally Distinct Mechanisms. Cell 161, 1058-1073

54. Kaygun $\mathrm{H}$ and Marzluff WF (2005) Regulated degradation of replication-dependent histone mRNAs requires both ATR and Upf1. Nat Struct Mol Biol 12, 794-800

55. Kim YK, Furic L, Desgroseillers L and Maquat LE (2005) Mammalian Staufen1 recruits Upf1 to specific mRNA 3'UTRs so as to elicit mRNA decay. Cell 120, 195-208

56. Chang CH, Curtis JD, Maggi LB Jr et al (2013) Posttranscriptional control of $\mathrm{T}$ cell effector function by aerobic glycolysis. Cell 153, 1239-1251

57. Kozak M (2006) Rethinking some mechanisms invoked to explain translational regulation in eukaryotes. Gene 382, $1-11$ 